\title{
The RNA-binding protein RBM47 is a novel regulator of cell fate decisions by transcriptionally controlling the p53-p21-axis
}

\author{
Claudia Radine $^{1} \cdot$ Dominik Peters $^{1} \cdot$ Alina Reese $^{1} \cdot$ Judith Neuwahl $^{1} \cdot$ Wilfried Budach $^{1} \cdot$ Reiner U. Jänicke $^{1}$. \\ Dennis Sohn ${ }^{1}$
}

Received: 17 October 2018 / Revised: 26 August 2019 / Accepted: 28 August 2019 / Published online: 11 September 2019

(c) The Author(s), under exclusive licence to ADMC Associazione Differenziamento e Morte Cellulare 2019

\begin{abstract}
In recent years it has become more and more apparent that the regulation of gene expression by RNA-binding proteins (RBPs) is of utmost importance for most cellular signaling pathways. RBPs control several aspects of RNA biogenesis including splicing, localization, stability, and translation efficiency. One of these RBPs is RBM47 that recently has been suggested to function as a tumor suppressor as it was shown to suppress breast and colon cancer progression. Here we demonstrate that RBM47 is an important regulator of basal and DNA damage-induced p53 and p21 ${ }^{\mathrm{WAF} 1 / \mathrm{CIP} 1}$ protein expression. Knockdown of RBM47 by siRNAs results in a strong reduction in p53 mRNA and protein levels due to an impaired p53 promoter activity. Accordingly, overexpression of Flag-RBM47 enhances p53 promoter activity demonstrating that RBM47 regulates p53 at the transcriptional level. By controlling p53, knockdown of RBM47 concomitantly decreases also p21 expression at the transcriptional level, driving irradiated carcinoma cell lines from different entities into cell death rather than into senescence. Thus, RBM47 represents a novel molecular switch of cell fate decisions that functions as a regulator of the p53/p21-signaling axis.
\end{abstract}

\section{Introduction}

The tumor suppressor p53 is classically activated after DNA damage and plays a central role in cell fate decisions by upregulating its transcriptional targets, such as the proapoptotic Bcl-2-family protein PUMA or the senescenceinducing CDK inhibitor p $21^{\mathrm{WAF} 1 / \mathrm{CIP} 1}$ [1]. In recent years it became clear that besides its direct transcriptional effects, p53 also strongly influences translational processes by regulating the expression of miRNAs (e.g., miR-34a) or RNAbinding proteins (RBPs, e.g., RNPC1) [2, 3]. These miRNAs

These authors contributed equally: Reiner U. Jänicke, Dennis Sohn

Edited by M. Oren

Supplementary information The online version of this article (https:// doi.org/10.1038/s41418-019-0414-6) contains supplementary material, which is available to authorized users.

Dennis Sohn

dennis.sohn@uni-duesseldorf.de

1 Laboratory of Molecular Radiooncology, Clinic and Policlinic for Radiation Therapy and Radiooncology, Medical Faculty, Heinrich-Heine-University, Düsseldorf, Germany and RBPs bind to their target mRNAs, in most cases in the $3^{\prime}$ UTR, regulating their localization, stability, and translation efficiency, thereby enhancing and diversifying the response mediated by activated p53 after DNA damage. On the other hand, expression of p53 itself as well as of its target genes is also heavily regulated by miRNAs and RBPs on the transcriptional and translational level [4-6]. This functions both as a negative feedback response as well as to establish basal cellular activation thresholds for the diverse p53-signaling pathways. By regulating expression of p53 and p21, RBPs modulate their activities in cellular processes including apoptosis and senescence, thereby acting in a tumor suppressive or promoting manner $[7,8]$.

One of those tumor-suppressing RBPs is the recently identified RBM47 protein (RNA-binding motif protein 47) that together with SYNCRIP (also known as hnRNP-Q), hnRNP-R, DND1 (DND microRNA-mediated repression inhibitor 1), A1CF (APOBEC1 complementation factor), and RBM46 belongs to the hnRNP-R/Q-paralog family. All these family members are functionally involved in most aspects of mRNA-related pathways. RBM47 itself participates in developmental processes, as in its absence zebrafish head formation is lost or strongly reduced due to defects in Wnt8a signaling [9]. Furthermore, most homozygous 
RBM47 knockout mice die during days 10-11 of gestation [10] and the few surviving RBM47-/- mice are significantly smaller than their wild-type counterparts unable to ever close the size gap in their lifetime [10]. On the molecular level, RBM47 has been reported to play an important role in alternative splicing $[11,12]$, the biogenesis of specific miRNAs [13], and as an essential co-factor of APOBEC1-mediated C-to-U RNA editing [14]. In addition, RBM47 also positively regulates IL-10 protein expression in regulatory B-cells [15]. In lung adenocarcinoma, RBM47 protein expression is repressed by TGF-beta, as RBM47 is required for the efficient generation of KEAP1 and Cull3 that antagonize TGF-beta-induced Nrf2 activation [16]. Consistent with such a tumor suppressor activity, RBM47 strongly inhibits tumor progression and metastasis and was found accordingly to be substantially downregulated during the epithelial-to-mesenchymal transition (EMT) in breast and colon cancer [12, 17].

Similar to RBM47, p53 was also found downregulated during EMT [18]. Furthermore, RBM47 was shown to associate with the ATM protein [19], which is one of the most apical sensors for DNA double strand breaks. In addition, RBM47 binds to the mRNA of the p53-modifiying ubiquitin ligase MDM4 regulating its alternative splicing and expression [12]. Together, these observations indicate that RBM47 may be an important regulator of DNA damage-induced p53 signaling. As the EMT can also be induced by radiotherapy and because in recent years more and more research has focused on RBPs controlling the p53-p21-axis [20-22], we herein analyzed more closely the role of RBM47 in p53-signaling pathways.

\section{Materials and methods}

\section{Cell lines, treatments, and reagents}

HCT116 cells and their p53-deficient counterparts (p53-/-) were cultured in McCoy's 5A GlutaMAXMedium (Gibco, Thermo Fisher Scientific, Waltham, MA, USA), whereas the breast cancer cell line MCF-7 [23] was cultured in RPMI-1640 GlutaMAX (Gibco). The head and neck squamous-cell carcinoma (HNSCC) line UM-SCC74A [24] was cultivated in DMEM high glucose (Gibco) and the colorectal adenocarcinoma cell line LoVo [25] in F12K-Medium (Gibco). All cell lines express wild-type p53 and exhibit a functional DNA damage-induced p53/p21signaling pathway. All media were supplemented with $10 \%$ FCS and $100 \mathrm{U} / \mathrm{ml}$ penicillin and $0.1 \mathrm{mg} / \mathrm{ml}$ streptomycin (all from Biochrom GmbH, Berlin, Germany). HCT116 and MCF-7 cells were authenticated by DNA fingerprinting (DSMZ, Braunschweig, Germany) and are regularly thawed from these authenticated lot charge as well as routinely tested for mycoplasma contamination with the Venor ${ }^{\oplus} \mathrm{GeM}$ detection kit (Minerva Biolabs, Berlin, Germany). Cells were gamma-irradiated with $20 \mathrm{~Gy}$ using the Gulmay RS225 irradiation system at $175 \mathrm{kV}$. The fluorogenic caspase-3 substrate DEVD-AMC (N-acetyl-Asp-Glu-ValAsp-aminomethylcoumarin) and the lipophilic and fluorogenic beta-galactosidase substrate $\mathrm{C}_{12}$-FDG (5-Dodecanoylaminofluorescein di-beta-D-galactopyranoside) were from Biomol (Hamburg, Germany) and Marker Gene Technologies (Eugene, OR, USA), respectively. Bafilomycin A1 (BafA1) was acquired from Alfa Aesar (Thermo Fisher GmbH, Karlsruhe, Germany). Propidium iodide (PI), etoposide, actinomycin D (ActD), MG-132, DTT, the protease inhibitors PMSF, aprotinin, leupeptin, and pepstatin as well as the phosphatase inhibitors sodium orthovanadate and sodium pyrophosphate were from Sigma-Aldrich (Merck KGaA, Darmstadt, Germany).

\section{Antibodies}

The RBM47 antibody (\#ab154176) was from Abcam (Cambridge, UK), whereas the beta-actin antibody (\#A5316) was obtained from Sigma-Aldrich. From Calbiochem (Bad Soden, Germany) we purchased the monoclonal p53 (Ab-6) antibody (\#OP43), whereas the p21 monoclonal antibody (\#556430) was from BD Biosciences (Heidelberg, Germany). For the nuclear run-on (NRO) assay a mouse monoclonal BrdUantibody (clone IIB5, \#SC-32323) from Santa Cruz and a corresponding IgG1 isotype control antibody (\#5415) from Cell Signaling was employed. The infrared fluorescencelabeled secondary antibodies were from Li-Cor Biosciences (Lincoln, Nebraska, USA).

\section{Plasmids}

For the luciferase-promoter studies, we used the pGL4vector system from Promega (Mannheim, Germany). The 356 bp p53 promoter sequence was amplified by PCR with the primers 5'-ATA GAG CTC GAT CCA GCT GAG AGC AAA CG-3' and 5'-TTA CTC GAG CTA GAC TTT TGA GAA GCT CAA A-3' using the plasmid pGL2-356bp-p53Prom [26] as a template and subsequently cloned after restriction with SacI and XhoI into the pGL4.10-vector. For the p21 promoter we amplified a $4.3 \mathrm{~kb}$ portion of the p21 promoter with the primers 5'-TTA GCT AGC TCT GAG TGC CTC GGT GCC TCG-3' and 5'-TTA GGT ACC TAT CTC CAT CAA GGC TTC TGC-3' using genomic DNA isolated from HCT116 wild-type cells as a template. After restriction with NheI and KpnI this PCR product was cloned into the pGL4.10-vector. The pCMV6-Flag-RBM47 and its corresponding empty vector were from Origene (Rockville, MD, USA), and the pcDNA3 plasmid containing Flag-p53alpha was kindly provided by F. Essmann [27]. All cloning 
PCRs were performed with the proofreading Phusion High Fidelity DNA polymerase (New England Biolabs, Frankfurt am Main, Germany).

\section{Preparation of cell extracts, western blotting, and fluorometric determination of caspase-3-like DEVDase activities}

Cells were lysed in NP40-buffer (1\% NP40, $150 \mathrm{mM} \mathrm{NaCl}$, $50 \mathrm{mM}$ Tris-HCl pH 7.4) supplemented with $1 \mathrm{mM}$ DTT, the protease inhibitors aprotinin, pepstatin, leupeptin (all 10 $\mu \mathrm{g} / \mathrm{ml})$, PMSF $(1 \mathrm{mM})$, and the phosphatase inhibitors sodium orthovanadate and sodium pyrophosphate (both 1 $\mathrm{mM})$. Protein concentrations were quantified via the Bradford protein assay (BioRad, München, Germany). Thirty micrograms cellular extracts per sample were used for size separation SDS-PAGE and subsequent western blotting on low-infrared-emitting PVDF membranes (Millipore, Schwalbach, Germany). After incubation with primary antibodies, secondary infrared fluorochrome-labeled antibodies were used to visualize the corresponding proteins. The membranes were scanned using the Li-Cor Odyssey imaging system and densitometrically analyzed with the Odyssey V3.0 software. Caspase-3-like DEVDase activities were fluorometrically measured in a 96-microplate well containing $50 \mu \mathrm{g}$ cellular extract together with $50 \mu \mathrm{M}$ AcDEVD-AMC and $10 \mathrm{mM}$ DTT in substrate buffer $(100 \mathrm{mM}$ HEPES pH 7.3, $100 \mathrm{mM} \mathrm{NaCl}, 10 \%$ sucrose, 0.1 CHAPS) at $37^{\circ} \mathrm{C}$ for $1-5 \mathrm{~h}$ using the Tecan M200 microplate reader (Mainz, Germany) and are presented as arbitrary units.

\section{Transfection of cells with siRNAs and/or plasmids}

Cells were transfected with ON-TARGET-plus siRNA SMART-pools (Dharmacon, Horizon Discovery, Cambridge, UK) and plasmids using DharmaFECT1 and DharmaFECT DUO, respectively, according to the manufacturer's instructions. As a control, nontargeting SMART-pools or the corresponding empty vector backbones were used. For siRNA experiments, cells were equally divided $24-48 \mathrm{~h}$ after transfection and afterwards used for further treatments or plasmid transfections. Following plasmid transfection, cells either underwent treatment after $8-24 \mathrm{~h}$ or they were analzyed directly $24 \mathrm{~h}$ after transfection without any treatment.

\section{Flow cytometric determination of senescence- associated beta-galactosidase activity}

To quantify cells demonstrating elevated senescenceassociated beta-galactosidase (SABG) activity, we modified a protocol from Debacq-Chainiaux et al. [28] and used the lipophilic and fluorogenic substrate $\mathrm{C}_{12}$-FDG to label SABG-active cells. In short, the supernatant of cells was transferred into separate reaction tubes and medium containing $100 \mathrm{nM}$ BafA1 was added to the remaining cells on the plate. The cells located in the supernatant were precipitated by centrifugation, resuspended in $100 \mathrm{nM}$ BafA1containing medium, and added back into their corresponding well. After a $1 \mathrm{~h}$ incubation at $37^{\circ} \mathrm{C}$, the SABG substrate $\mathrm{C}_{12}$-FDG was added in a final concentration of $33 \mu \mathrm{M}$ and incubation at $37^{\circ} \mathrm{C}$ was continued for $2-3 \mathrm{~h}$. Subsequently, the supernatant was transferred into a FACS tube, and the remaining adherent cells were harvested by trypsinization and merged with its respective supernatant. After two washes with PBS, the cells were resuspended in PBS and quantified for elevated FITC-fluorescence (that indicates SABG activity) using a LSR-Fortessa (BD Biosciences) and the FACSDiva analysis software. For each determination, a minimum of 10,000 cells was analyzed.

\section{Flow cytometric determination of cell death by propidium iodide (PI) uptake}

After treatment, supernatants were transferred into FACS tubes and the remaining adherent cells were harvested by trypsinization and merged together with their respective supernatants. After $15 \mathrm{~min}$ on ice to allow the cells to recover from the trypsinization stress, cells were precipitated by centrifugation and the pellets were directly resuspended in PI-buffer $(2.5 \mu \mathrm{g} / \mathrm{ml}$ PI in PBS). After an additional $10 \mathrm{~min}$ on ice, the cells were analyzed using a LSR-Fortessa (BD Biosciences) and the FACSDiva analysis software. The living, PI-negative cell population was quantified using a dot plot displaying the forward scatter and PI-fluorescence intensity. For each determination, a minimum of 10,000 cells was analyzed.

\section{Quantification of cell death by measuring lactate dehydrogenase (LDH) activity in the cell culture supernatant}

To quantify LDH activity in the supernatant of cells we used the cytotoxicity detection kit (LDH) from Roche (Mannheim, Germany) according to the manufacturer's instructions. In short, cell-free culture supernatants were harvested and a $100 \mu \mathrm{l}$ aliquot of each was mixed in a 96-well microtiter plate with an equal amount of the reaction mixture from the kit. LDH activity was subsequently quantified by measuring the absorbance at $490 \mathrm{~nm}$ in $3 \mathrm{~min}$ intervals for $30 \mathrm{~min}$ at room temperature in a Tecan M200 microplate reader.

\section{RNA isolation, Real-Time-PCR, and mRNA stability assay}

Total RNA was isolated using the RNeasy Mini kit from Qiagen (Hilden, Germany) according to the manufacturer's 
description. One microgram total RNA was transcribed into cDNA with the High-Capacity cDNA reverse transcription kit (Thermo Fisher Scientific) and subsequently analyzed using specific Taqman probes for p21, p53, PUMA, and RSK1 mRNA (Thermo Fisher Scientific) and actin or GAPDH probes as endogenous controls. Quantitative RealTime-PCR was carried out with an ABI 7300 Real-TimePCR system (Thermo Fisher Scientific). Relative mRNA expression levels of treated cells compared to untreated control cells were calculated by the $2^{\Delta(\Delta \mathrm{Ct})}$ method. For the mRNA stability assay, $1 \mu \mathrm{g} / \mathrm{ml}$ ActD was added to the samples $2 \mathrm{~h}$ after treatment with $\gamma$ IR. Total RNA was isolated at different time points after ActD addition and quantitative Real-Time-PCR was carried out as described above. The time-dependent decrease in mRNA expression levels after ActD addition was used to establish a regression line and to calculate the half-life of the mRNA at the moment of ActD addition.

\section{Nuclear run-on assay with BrU labeling of actively transcribed RNAs}

To quantify nascent transcription we performed an NRO assay followed by Real-Time-PCR using a slightly modified protocol from Roberts et al. [29]. In short, we isolated nuclei from 2 million cells that were either transfected with a control siRNA or an siRNA directed against RBM47 3 days before harvest. Subsequently the NRO was performed for $30 \mathrm{~min}$ at $30^{\circ} \mathrm{C}$ in the presence of $\mathrm{BrU}(5-$ Bromouridine, Cayman Chemical, Ann Harbor, MI, USA). Genomic DNA was removed afterwards by digestion using the RNase-free DNase Set (Qiagen). The nuclear RNA was purified using the RNeasy Mini Kit (Qiagen) and the actively transcribed bromouridylated RNA was isolated out of $5 \mu \mathrm{g}$ of nuclear RNA by immunoprecipitation with protein $\mathrm{G}$ sepharose beads using a BrdU-specific antibody (Cell Signaling). To ensure the specific enrichment of BrUlabeled NRO-RNAs, we also quantified background binding of RNAs by using an isotype control antibody in the IP. The bound RNA was isolated by using TRI-Reagent (Sigma-Aldrich) and transcribed into cDNA using the High-Capacity cDNA Kit (Life Technologies). The enrichment of BrU-labeled NRO-RNAs was analyzed by SYBR-Green Real-Time-PCR using the Luna Universal qPCR Master Mix (New England Biolabs) with specific primer pairs for GAPDH, actin, p21 (sequences from [29]), and p53 exon 1 (p53_Exon1_fw: 5'-AAA GTC TAG AGC CAC CGT CC-3'; p53_Exon1_rev: 5'-GTC ACC GTC GTG GAA AGC A-3') and the ABI 7300 Real-TimePCR system (Life Technologies). The amounts of BrUlabeled actin, p21, or p53 RNAs were quantified by using the $2^{(\Delta(\Delta \mathrm{Ct}))}$ method and the corresponding GAPDH values as a normalization factor.

\section{Determination of p53 and p21 promoter-dependent luciferase activities}

For all luciferase activity assays, the Dual-luciferase-reporter system from Promega was employed and the experiments were performed as described before [21]. In short, analyses of p53- or p21-promoter-dependent luciferase activities were performed by transfecting HCT116 cells with the promotercontaining pGL4.10 firefly luciferase plasmids or with the promoterless pGL4.10-vector as a negative control. To take varying transfection efficiencies into account, the renilla luciferase-encoding plasmid pGL4.74 was cotransfected as a normalization control. To analyze the effect of RBM47 on the p53- or the p21-promoter-driven luciferase activities, siRNAs directed against RBM47 or the pCMV6-Flag-RBM47 plasmid were additionally transfected. After $24 \mathrm{~h}$, cells were equally divided into two wells and either gamma-irradiated or left untreated. After another $24 \mathrm{~h}$, cells were harvested and the luciferase activities were determined with the Dual-luciferasereporter system (Promega) according to the manufacturer's instructions in a Centro LB960 microplate luminometer (Berthold Technologies, Bad Wildbad, Germany).

\section{Statistical analyses}

For all statistical analyses a paired (if applicable) student's $t$-test was performed. ${ }^{*} p<0.05, * * p<0.01, * * * p<0.001$.

\section{Results}

\section{RBM47 positively controls expression of p53 at the transcriptional level}

As RBM47 and p53 were both found to be downregulated during EMT [12, 17, 18], we addressed the question of whether expression of these proteins is interconnected in some way. For this purpose we first analyzed their expression levels in p53-proficient (wild type) and p53deficient (p53-/-) HCT116 colon carcinoma cells that have been either left untreated or exposed to ionizing irradiation $(\gamma \mathrm{IR})$ or etoposide. Although both DNA-damaging insults induced in HCT116 wild-type cells a time-dependent accumulation of p53 together with a vast increase in its target protein p21, expression of RBM47 was not affected (Fig. 1a, b). Though RBM47 levels appeared slightly elevated in irradiated wild-type cells when compared with similar treated p53-deficient cells, suggesting a p53dependent regulation mechanism, densitometric analyses failed to verify this assumption (data not shown). Furthermore, as RBM47 protein levels in untreated p53-deficient HCT116 cells were indistinguishable from those in wildtype cells, these results suggest that expression of RBM47 
A

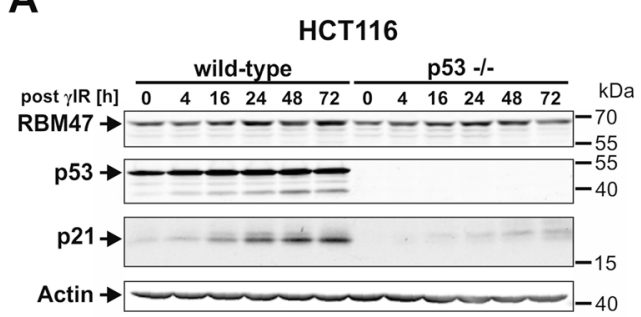

C

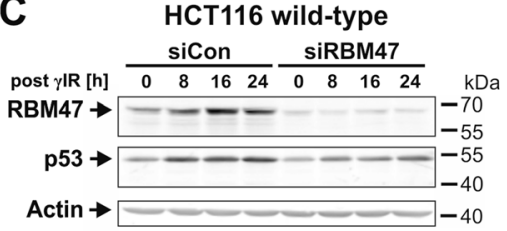

E

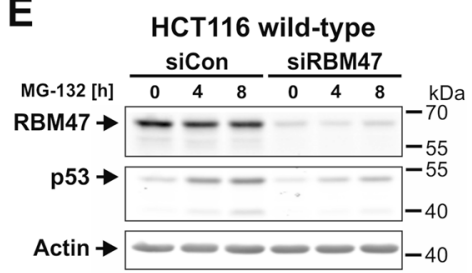

Fig. 1 Knockdown of RBM47 inhibits p53 protein expression. a, b p53-proficient (wildtype) and p53-deficient (p53-/-) HCT116 cells were exposed to $\gamma \mathrm{IR}$ (a) or etoposide (b) and analyzed after the indicated times for expression of RBM47, p53, p21, and, as a loading control, for the status of actin. c-f After a preceding transfection ( $48 \mathrm{~h})$ with either the control siRNA (siCon) or the RBM47 siRNA

is most likely not controlled by p53. In contrast, when RBM47 was downregulated by a specific siRNA, p53 protein expression in wild-type cells was consistently impaired under control conditions as well as in response to $\gamma$ IR or etoposide (Fig. 1c, d; Supplementary Fig. 1A). This effect was also observed in the HNSCC line UM-SCC-74A (Supplementary Fig. 1B), indicating that RBM47 may be generally required for the upregulation of p53 expression under control and stress conditions.

p53 protein levels are mainly controlled by posttranslational modifications that regulate its degradation by the proteasome [30]. Coincidently, RBM47 was reported to control expression of MDM4 (also known as MDMX) [12], that together with its homolog MDM2 [31] regulates p53 ubiquitination and subsequent degradation. To determine whether RBM47 controls p53 expression at the posttranslational level, HCT116 wild-type cells were transfected with either a control siRNA or the RBM47-specific siRNA followed by an incubation with the proteasomal inhibitor MG-132. This treatment resulted in the expected stabilization of p53 regardless of whether RBM47 was present or not (Fig. 1e). However, overall accumulation of p53 protein levels in response to MG-132 remained dramatically reduced in RBM47-depleted cells compared with MG-132-
B
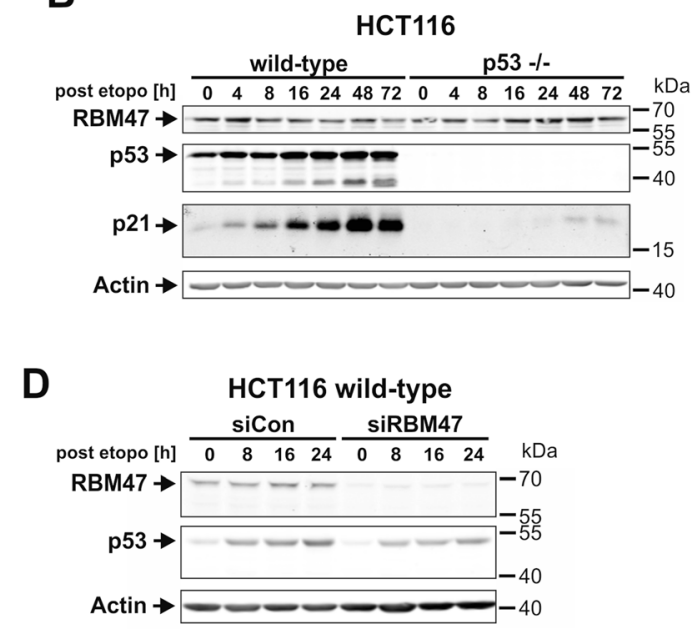

$\mathbf{F}$

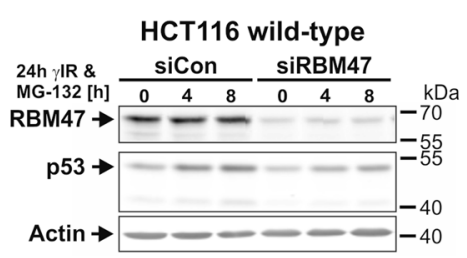

(siRBM47) HCT116 wild-type cells were exposed to $\gamma$ IR (c), etoposide (d), MG-132 (e), or $\gamma$ IR together with MG-132 (f) and analyzed at the indicated times for expression of RBM47, p53, and actin. Blots shown are representative blots of at least three independent experiments

treated cells transfected with the control siRNA (Fig. 1e). Also an additional exposure to $\gamma$ IR in the presence of MG132 could not increase the $\mathrm{p} 53$ stabilization rate following knockdown of RBM47 (Fig. 1f). Noteworthy, densitometric analyses demonstrate that although the accumulation of p53 protein in response to MG-132 was much less pronounced in RBM47-depleted cells compared with similarly treated cells transfected with the control siRNA, their timeand MG-132-dependent p53 stabilization rates progressed identically (Supplementary Fig. 1C). Together, these data strongly imply that RBM47 controls p53 expression at the transcriptional or translational level independently of such a posttranslational stabilization mechanism.

Consistent with this conclusion, p53 mRNA levels were found considerably repressed following loss of RBM47 not only in HCT116 wild-type cells (Fig. 2a), but also in UMSCC-74A, MCF-7 and, albeit to a smaller extent, in LoVo cells (Supplementary Fig 2A, C, E). In contrast, abundance of the p90 ribosomal S6 kinase 1 (RSK1) mRNA remained unaffected following RBM47 depletion (Supplementary Fig. 1D), suggesting that RBM47 specifically controls expression of the p53 mRNA. Interestingly, p53 mRNA suppression in consequence of a RBM47 knockdown was evident regardless of whether or not HCT116 wild-type 


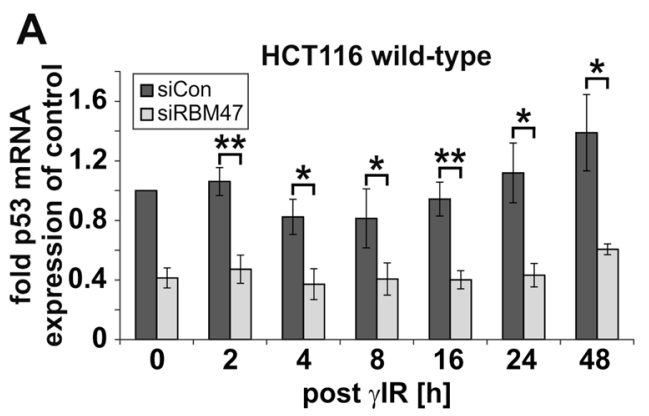

B
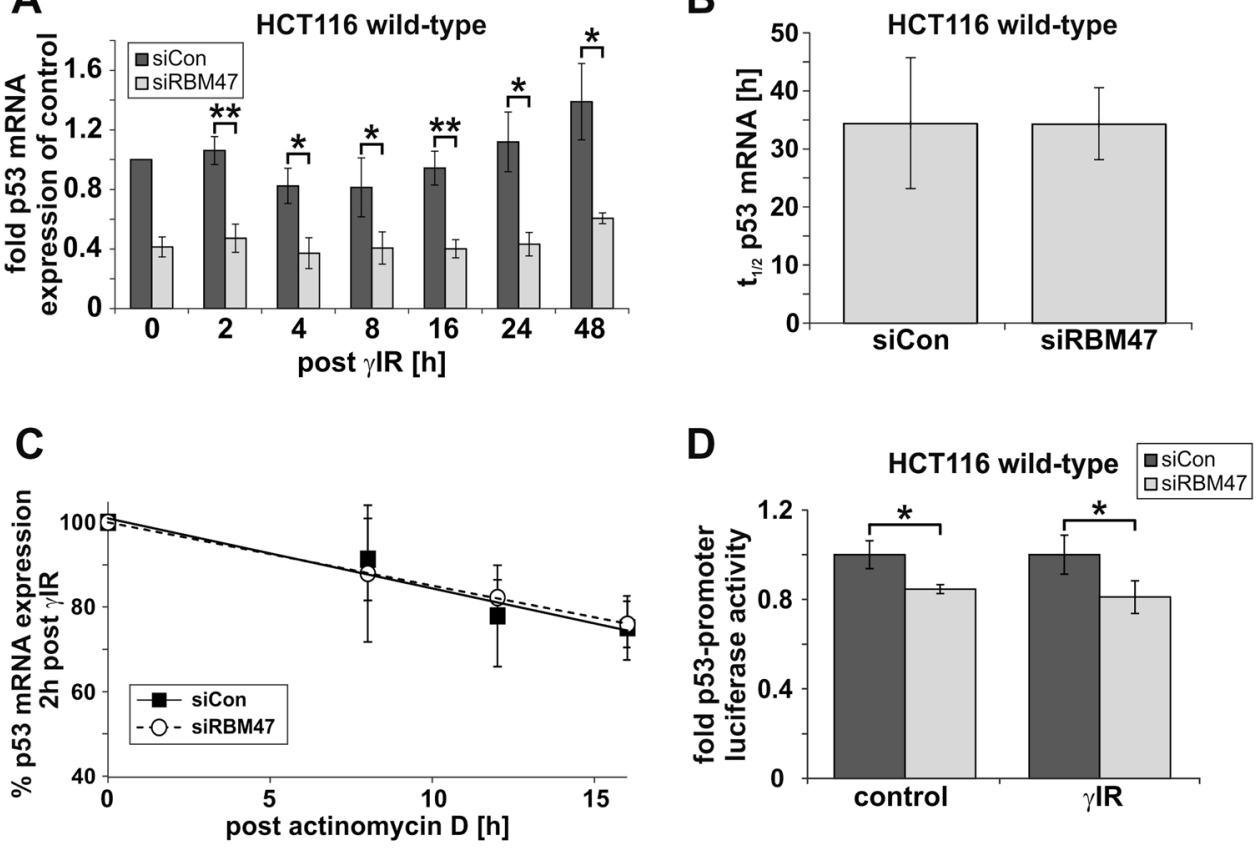

$\mathbf{E}$

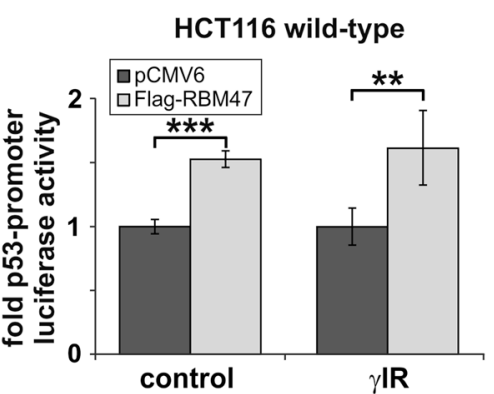

$\mathbf{F}$

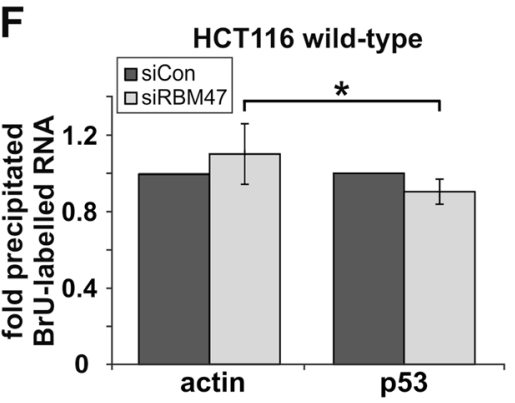

Fig. 2 RBM47 controls p53 expression at the transcriptional level. a-c Quantitative Real-Time-PCR for the determination of p53 mRNA expression levels in HCT116 wild-type cells that were transfected $48 \mathrm{~h}$ before irradiation with either the control siRNA or the RBM47 siRNA. For (a) the total RNA was isolated at the indicated times, whereas cells in (b) and (c) were treated $2 \mathrm{~h}$ following their exposure to $\gamma \mathrm{IR}$ with actinomycin D for additional $0,8,12$, and $16 \mathrm{~h}$. RNA was analyzed with transcript-specific probes from Life Technologies. d, e Determination of luciferase activities in HCT116 wild-type cells $24 \mathrm{~h}$ post exposure to $\gamma \mathrm{IR}$. Cells were cotransfected with either the RBM47 siRNA (d) or a Flag-RBM47 construct (e) together with a

cells were exposed to DNA-damaging insults, implying that RBM47 is required for an efficient p53 mRNA expression under control and stress conditions (Fig. 2a). Further investigations into this matter revealed that loss of RBM47 does not affect stability of the p53 mRNA (Fig. 2b, c), strongly indicating that RBM47 interferes with p53 expression at the transcriptional level. Indeed, performing reporter gene assays, we found that knockdown of RBM47 impaired expression of the luciferase gene under the control of a $356 \mathrm{bp}$ p53 promoter fragment both in untreated and irradiated HCT116 wild-type cells (Fig. 2d). Consistently, vector expressing the luciferase gene downstream of the $356 \mathrm{bp}$ p53 promoter. $\mathbf{f}$ Determination of the transcriptional rate by performing a nuclear run-on assay in HCT116 wild-type cells $72 \mathrm{~h}$ post transfection with the control siRNA or the RBM47 siRNA. Nuclei were isolated and actively transcribed RNAs were labeled with $\mathrm{BrU}$ in a nuclear runon assay. After purification by immunoprecipitation, BrU-labeled RNAs were quantified by SYBR-Green Real Time-PCR using specific primer pairs for GAPDH, actin, and p53 exon 1. Shown is the amount of precipitated BrU-labeled actin and p53 RNAs normalized to the corresponding GAPDH RNAs. Values shown in (a-f) are the mean of at least three independent experiments \pm S.D exogenously transfected Flag-RBM47 increased p53 promoter-driven luciferase expression also under both conditions (Fig. 2e). To further verify that RBM47 controls p53 promoter activity, we also performed an NRO assay. Newly transcribed RNAs from isolated nuclei out of cells transfected with a control siRNA or an siRNA directed against RBM47 were labeled with $\mathrm{BrU}$ and subsequently purified by immunoprecipitation. Real-Time-PCR analyses quantifying these mRNAs demonstrated that the actual transcription rate of the p53 gene is reduced in the absence of RBM47 (Fig. 2f). As the nuclei were isolated from 
Fig. 3 RBM47 controls p21 expression indirectly in a p53dependent manner. a, b After a preceding transfection $(48 \mathrm{~h})$ with either the control siRNA (siCon) or the RBM47 siRNA (siRBM47) HCT116 wild-type cells were exposed to $\gamma$ IR (a) or etoposide (b) and analyzed at the indicated times for expression of RBM47, p53, p21, and actin. Blots shown are representative blots of at least three independent experiments. c-e Quantitative Real-TimePCR for the determination of p21 (c, e) and Puma (d) mRNA expression levels in p53proficient (wild-type) $(\mathbf{c}, \mathbf{d})$ and p53-deficient (p53-/-) HCT116 cells (e). Cells were transfected with either the control siRNA or the RBM47 siRNA $48 \mathrm{~h}$ before they were irradiated. Total RNA was isolated 0,4 , and $24 \mathrm{~h}$ following their exposure to $\gamma \mathrm{IR}$ and analyzed with transcript-specific probes from Life Technologies. Values shown in $(\mathbf{c}-\mathbf{e})$ are the mean of at least three independent experiments \pm S.D
A

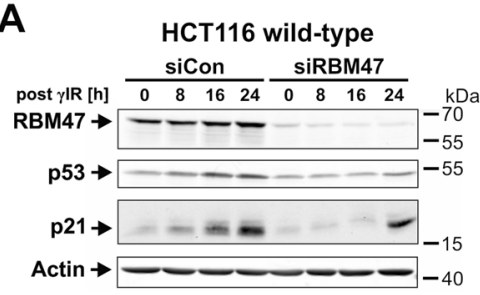

C

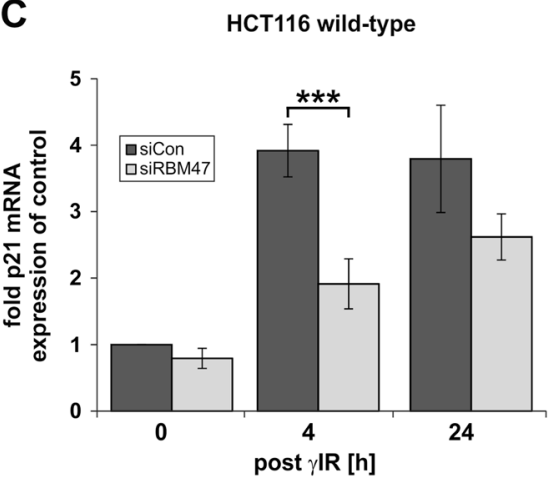

E

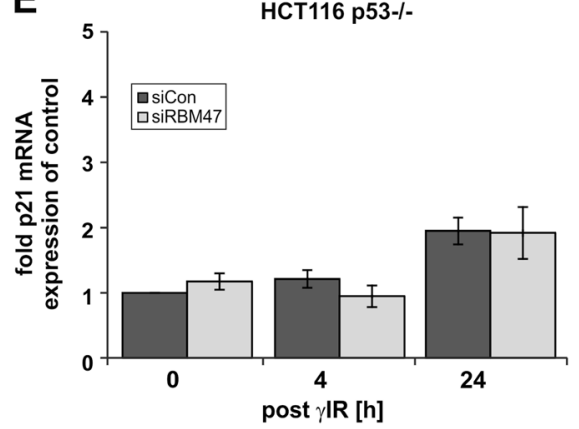

B
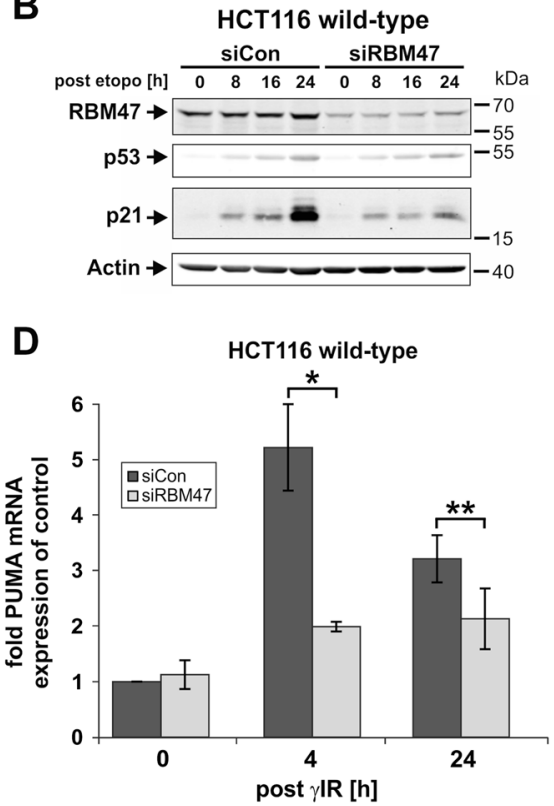

unstimulated cells, these data further support our former conclusion that RBM47 positively regulates p53 expression at the transcriptional level even under basal conditions independently of a stress stimulus.

\section{RBM47 controls expression of p21 indirectly through regulation of p53 promoter activity}

Next, we addressed the question of whether expression of $\mathrm{p} 21^{\mathrm{WAF} 1 / \mathrm{CIP} 1}$, a prominent $\mathrm{p} 53$ target, is also affected by RBM47. As expected, in accordance with an impaired p53 expression, knockdown of RBM47 also inhibited protein expression of this cyclin-dependent kinase inhibitor following exposure of HCT116 wild-type cells to $\gamma \mathrm{IR}$ or etoposide (Fig. 3a, b). Real-Time-PCR analyses revealed that the diminished p21 protein levels observed in HCT116 wild-type cells in the absence of RBM47 are due to a decreased p21 mRNA expression (Fig. 3c). The reduction of p21 mRNA levels after knockdown of RBM47 was also evident in UMSCC-74A, MCF-7 and again to a smaller extent in LoVo cells
(Supplementary Fig 2B, D, F). Knockdown of RBM47 severely impaired also expression of another prominent p53 target gene, namely Puma (Fig. 3d), suggesting that both events are caused by a decreased p53 expression. Indeed, knockdown of RBM47 in p53-deficient HCT116 cells did not affect p21 mRNA expression (Fig. 3e). Consistently, loss of RBM47 in HCT116 wild-type cells strongly inhibited expression of the luciferase gene that is under the control of a $4 \mathrm{~kb}$ p21 promoter fragment (Fig. 4a), whereas overexpression of Flag-RBM47 resulted in increased luciferase activities (Fig. 4b). Again, p21 promoter-driven luciferase activities remained unaffected when RBM47 was depleted or overexpressed in p53-deficient HCT116 cells (Fig. 4a, b). Furthermore, knockdown of RBM47 was unable to inhibit p21 promoter-driven luciferase activities when these were induced in p53-deficient HCT116 cells in response to an exogenously transfected Flag-p53 construct that is expressed under the control of the CMV promoter instead of the natural p53 promoter (Fig. 4c). Finally, as an NRO assay also revealed a reduced $\mathrm{p} 21$ transcription rate in the absence of 
A

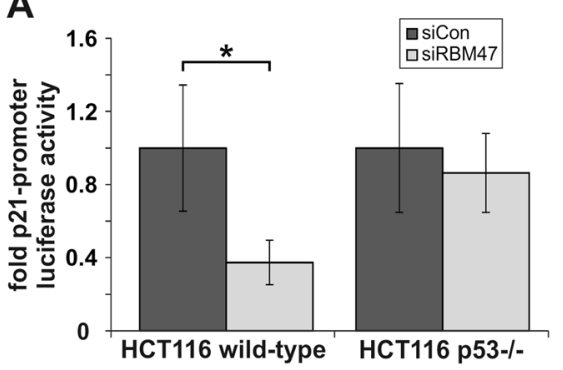

C

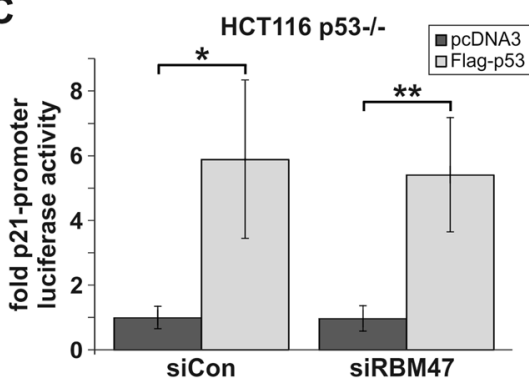

B

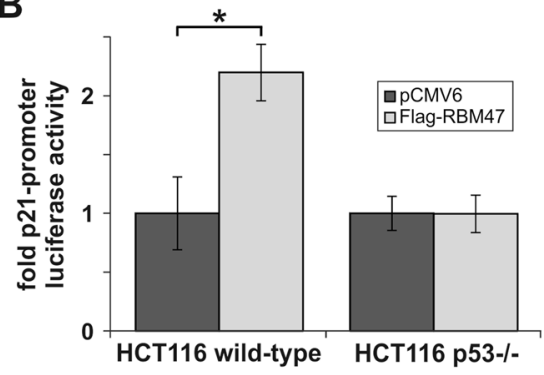

D

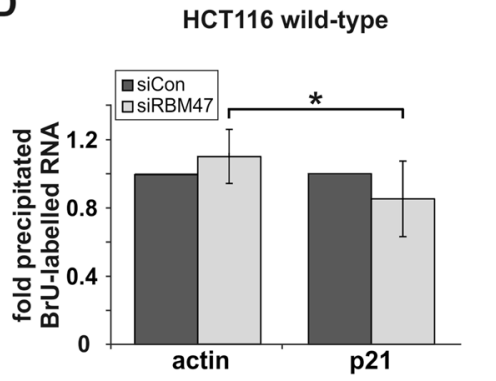

Fig. 4 RBM47 controls p21 promoter activity indirectly in a p53dependent manner. a-c Determination of luciferase activities in HCT116 wild-type and p53-deficient cells $24 \mathrm{~h}$ post transfection with either the RBM47 siRNA (a), a Flag-RBM47 construct (b), or a Flagp53 expression plasmid (c) together with a vector expressing the luciferase gene downstream of the $4.3 \mathrm{~kb}$ p21 promoter fragment. d Determination of the transcriptional rate by performing a nuclear runon assay in HCT116 wild-type cells $72 \mathrm{~h}$ post transfection with the control siRNA or the RBM47 siRNA. Nuclei were isolated and

RBM47 (Fig. 4d), our results clearly demonstrate that RBM47 controls p21 expression indirectly at the transcriptional level by regulating endogenous p53 promoter activity.

\section{The biological function of RBM47}

To finally address the biological consequences of our findings, we made use of the HCT116 wild-type cells that in response to $\gamma \mathrm{IR}$ mainly undergo a permanent cell cycle arrest (senescence) rather than apoptosis [32]. This is due to the p53-dependent expression of $\mathrm{p} 21$ that in addition to its role as an antiproliferative molecule functions in these cells also in an antiapoptotic manner [32, 33]. In contrast, HCT116 cells deficient in p53 undergo apoptosis because they are unable to upregulate p21 expression in response to DNA damage. Flow cytometric quantification of HCT116 wildtype cells positive for SABG activity revealed that the knockdown of RBM47 resulted in a substantial decrease of senescent cells in response to $\gamma$ IR (Fig. 5a). In accordance with such a suppressive effect on the appearance of senescent cells, RBM47 depletion not only caused an increase in cell death as measured by the uptake of PI and the release of LDH activity (Fig. 5b, c), but, in addition, also in elevated caspase-3-like DEVDase activities, both under control and stress $(\gamma \mathrm{IR})$ conditions (Fig. 5d). As all these events actively transcribed RNAs were labeled with $\mathrm{BrU}$ in a nuclear run-on assay. After purification by immunoprecipitation, BrU-labeled RNAs were quantified by SYBR-Green Real Time-PCR using specific primer pairs for GAPDH, actin and p21. Shown is the amount of precipitated BrU-labeled actin and p21 RNAs. Please note that these experiments were performed together with the experiments shown in Fig. 2f, requiring the repeated usage of the actin control data. Values shown are the mean of three $(\mathbf{a}, \mathbf{b})$ and five $(\mathbf{c}, \mathbf{d})$ independent experiments \pm S.D

remained unaltered in p53-deficient cells following loss of RBM47 (Fig. 5b, c, e), our results demonstrate that the RBM47-mediated impact on the cellular fate of HCT116 is mainly driven by promoting the p53-dependent p21 expression pathway. Furthermore, the observation that the absence of RBM47 causes a similar shift from IR-induced senescence (SABG) to cell death (PI uptake) in both UMSCC-74A (Supplementary Fig 3A, B) and MCF-7 (Supplementary Fig 3C, D) cells indicates that this function of RBM47 is not confined to one cell lineage or tumor entity, but may represent a general regulatory mechanism.

\section{Discussion}

In recent years the regulation of gene expression by RBPs has moved into the center of attention [7, 8]. More and more RBPs have been identified that intervene in the control of central cellular signaling pathways including cell fate decisions. Here we demonstrate that the novel RBP RBM47 acts as an important regulator of the p53-p21 axis. Expression of the $\mathrm{p} 53$ protein is strongly impaired in the absence of RBM47 both under basal conditions as well as after DNA damage induced by radio- or chemotherapy. Several pieces of evidence including the finding that 
Fig. 5 RBM47 modulates the fate of irradiated HCT116 cells in a p53-dependent manner. HCT116 wild type (a-d) and p53-deficient cells $(\mathbf{b}, \mathbf{c}, \mathbf{e})$ were transfected $48 \mathrm{~h}$ before $\gamma$ IR exposure with either the control siRNA (siCon) or the RBM47 siRNA (siRBM47). For senescence assessment (SABG activity) (a) and the analysis of PI uptake (b), cells were analyzed cytometrically $72 \mathrm{~h}$ post $\gamma \mathrm{IR}$, whereas the release of LDH into the supernatant (c) was quantified at day 5 post treatment. Apoptosis induction (d, e) was determined by the evaluation of caspase-3-like DEVDase activities at 0,24, and $48 \mathrm{~h}$ post $\gamma \mathrm{IR}$. Values shown are the mean of at least three independent experiments \pm S.D
A

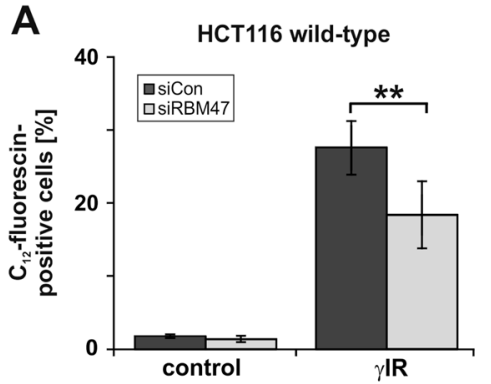

C

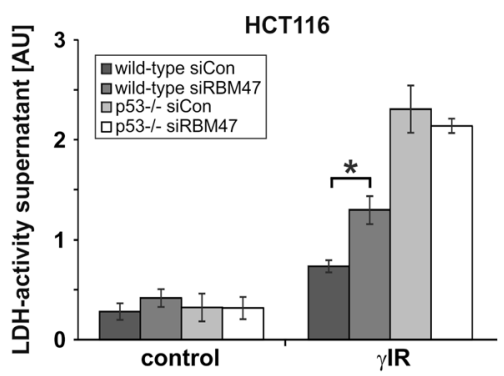

E

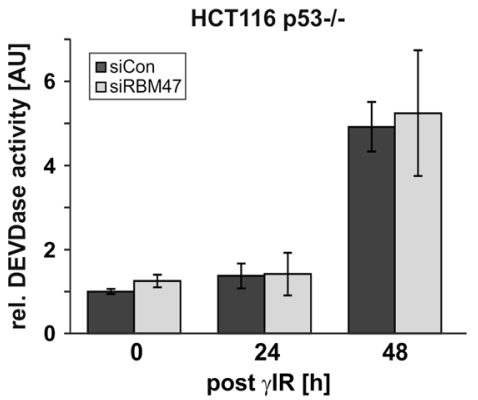

B

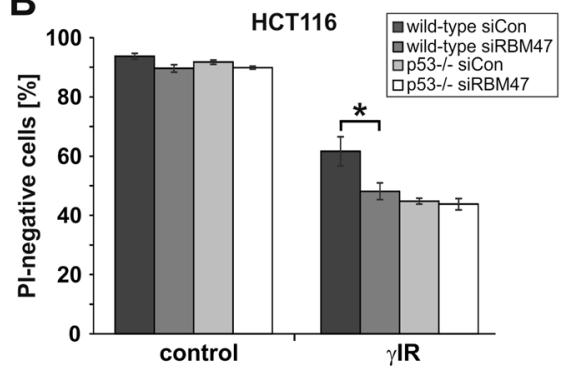

D

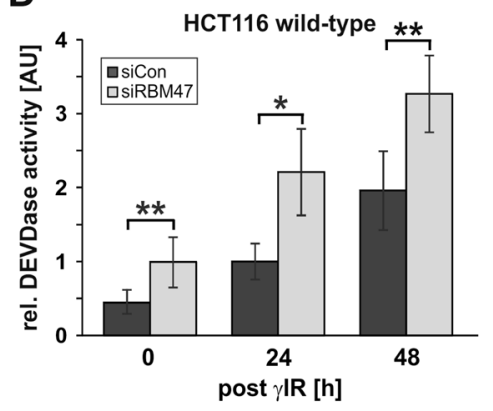

RBM47 targets the mRNA of MDM4 [12] that in concert with its homolog, the ubiquitin ligase MDM2 [31], regulates p53 protein stability, suggested that RBM47 might be required for posttranslational stabilization of p53. Supporting this assumption is also the recently reported interaction of RBM47 with the DNA damage sensor kinase ATM [19] that controls p53 protein stability by phosphorylating MDM2 and p53 itself. However, inhibition of the proteasome by MG-132 could not restore p53 protein expression up to control levels following knockdown of RBM47, strongly arguing against such a posttranslational control mechanism. As the absence of RBM47 also failed to alter the stability of the p53 mRNA and instead caused a decrease in $\mathrm{p} 53$ promoter activity resulting in a reduced $\mathrm{p} 53$ mRNA production, our data obtained from several cell lines originating from different tumor types including colon (HCT116, LoVo), HNSCC (UM-SCC-74A), and breast carcinoma (MCF-7) demonstrate that RBM47 is a common regulator of p53 expression at the transcriptional level.

The underlying mechanism of RBM47 regulating p53 promoter activity, however, remains unknown. RBM47 may either activate a transcriptional activator/co-activator or repress an inhibitor of the p53 promoter, most likely by regulating their respective mRNAs. As both RBM47 and p53 are inhibitors of the EMT [12, 17, 18], it is tempting to speculate that the transcriptional activators of this program such as TWIST1/2, ZEB1/2, or SNAI1/2 that also function as transcriptional repressors of p53 [18] may be regulated by RBM47. Unfortunately, based on the data sets available so far, none of these EMT factors are found to be associated with RBM47, neither at the protein (BioGrid and IntAct protein-protein interaction databases) or mRNA level $[12,16]$. Also other known regulators of p53 promoter activity [34] have so far not been described to be RBM47 targets with the exception of the ETS1 mRNA that was together with Flag-RBM47 precipitated from A549 cells as one of numerous targets (rank 229) [12]. Unfortunately, this tumor suppressor transcription factor and positive regulator of p53 promoter activity [35] is not expressed in HCT116 cells [36]. Thus, it cannot be responsible for the reduction of the p53 mRNA we observed in these cells in the absence of RBM47.

Although two intensive screening efforts have been undertaken to identify mRNA targets of RBM47 and to 
determine its consensus binding sequence [12, 16, 37], these analyses did not lead to the identification of potential candidates known to transcriptionally regulate p53 expression. With regard to this, it is noteworthy that both screening attempts produced almost no overlapping hits and mainly identified totally different mRNAs as binding partners of RBM47. Naturally, this could be due to the different cellular systems employed and the readouts used, or it may just demonstrate the versatility of the RBM47 mRNA binding capabilities. Indeed, besides an extremely vague polyU sequence, Vanharanta et al. [12] were unable to determine a precise mRNA binding consensus sequence for RBM47 and suggested that this is due to its broad RNAbinding proficiency. On the other hand, Ray et al. [37] used a synthetic RNA library to determine the consensus sequence of various RBPs in a systemic approach and identified GAUGAU as the most preferred binding site for RBM47. The reason for these discrepant findings is presently unknown, but may be due to the source of RBM47 used (human [12] vs chicken/frog [37]).

Because RBM47 is localized to both the cytoplasm and the nucleus $[9,16]$, we cannot completely exclude a direct effect of RBM47 on p53 promoter activity, e.g., by binding to long noncoding RNAs (lncRNAs) that are responsible for $\mathrm{p} 53$ promoter methylation. So far, several lncRNAs have been identified that regulate p53 expression by different mechanisms [38]. Two of them, MALAT1 [39] and HOTAIR [40], were shown to induce p53 promoter methylation thereby reducing promoter activity and p53 mRNA production. Although these two lncRNAs could likely represent potential targets of RBM47, it is so far unknown whether RBM47 binds to these lncRNAs and whether this would modulate their stability and/or activity.

Regarding its biological significance, RBM47 exhibits already multiple functions solely by regulating expression of the similarly versatile p53 protein. The tumor suppressor p53 regulates numerous complex signaling pathways $[1,41]$ not only following its activation-induced accumulation but also at basal levels, e.g., by controlling the baseline expression of several important tumor suppressor genes [42]. Consequently, RBM47 controls p53-dependent cell fate decisions, e.g., the herein analyzed scenario of a p21mediated senescence induction following DNA damage. It is easily imaginable that in other cellular systems in which p53 exerts other, totally different activities (e.g., regulating autophagy, inducing apoptosis, and changing metabolism), the presence and activity of RBM47 will surely control also these pathways. Another interesting aspect that is very tempting to speculate about is that RBM47 might even represent a protumorigenic factor in certain cells by promoting expression of p53 gain-of-function mutants. Such a possible and fascinating interplay of RBM47 and p53 in cancer formation should be analyzed in more detail in future studies.

We also have to keep in mind that based on its involvement in numerous aspects of RNA metabolism, such as mRNA splicing, translation, stability, and editing, RBM47 also controls expression of other proteins besides p53 [12, 14-16, 43]. These could be p53 downstream targets (e.g., p21, PUMA, or others) and/or proteins acting independently of p53 signaling enabling RBM47 to fine tune cell fate decisions in an extremely complex and may be even opposing manner. Unfortunately, not many RBM47 targets have been identified so far. For example, RBM47 was reported to indirectly repress SMAD3mediated transcription of p21 and MAFK, while positively controlling translation of KEAP1 and Cullin 3 mRNAs that belong to an E3 ubiquitin ligase complex [16]. As all of these components differently impact on the activation of the transcription factor Nrf2 that is associated with poor prognosis and resistance to chemoand radiotherapy [44], these observations further illustrate the complex nature of RBM47-mediated signaling. RBM47 was also reported to interact with the mRNA of IKB-alpha [16] that controls signaling of the antiapoptotic NFKB transcription factor. However, the biological significance of this interaction was not investigated in this study.

Taken together, by facilitating transcription of the p53 gene and its target $\mathrm{p} 21$, our study strongly supports a role for RBM47 as an important tumor suppressor. For future research endeavors aiming at further elucidating these and other cellular functions of RBM47, it is absolutely necessary to reliably identify additional mRNA targets of RBM47 as well as to decipher its consensus RNA-binding sequence. In addition, it will be important to verify our conclusion in a more physiological setting, e.g., in xenograft or other mouse models where RBM47proficient or -deficient tumors are exposed to radio- or chemotherapy. Together, such information will be extremely useful for the development of future anticancer strategies.

Acknowledgements We thank C. Disselhoff and S. Dangeleit for excellent technical assistance and Bert Vogelstein for providing the two HCT116 cell lines, Nikolas Stoecklein for the LoVo cells and Kathrin Scheckenbach for the UM-SCC-74A cells.

Funding This work was supported by grants from the Deutsche Forschungsgemeinschaft to RUJ (JA 1060/3-1; 1060/5-1) and to DS (SO $881 / 5-1)$, and from the medical research council of the University Düsseldorf to RUJ.

\section{Compliance with ethical standards}

Conflict of interest The authors declare that they have no conflict of interest. 
Publisher's note Springer Nature remains neutral with regard to jurisdictional claims in published maps and institutional affiliations.

\section{References}

1. Kastenhuber ER, Lowe SW. Putting p53 in context. Cell. 2017; 170:1062-78.

2. Marcel V, Catez F, Diaz JJ. p53, a translational regulator: contribution to its tumour-suppressor activity. Oncogene. 2015;34:5513-23.

3. Zaccara S, Tebaldi T, Pederiva C, Ciribilli Y, Bisio A, Inga A. p53-directed translational control can shape and expand the universe of p53 target genes. Cell Death Differ. 2014;21: 1522-34.

4. Reinhardt HC, Cannell IG, Morandell S, Yaffe MB. Is posttranscriptional stabilization, splicing and translation of selective mRNAs a key to the DNA damage response? Cell Cycle. 2011;10:23-7.

5. Sohn D, Peters D, Piekorz RP, Budach W, Jänicke RU. miR-30e controls DNA damage-induced stress responses by modulating expression of the CDK inhibitor p21WAF1/CIP1 and caspase-3. Oncotarget. 2016;7:15915-29.

6. Zhang J, Chen X. Posttranscriptional regulation of p53 and its targets by RNA-binding proteins. Curr Mol Med. 2008;8:845-9.

7. Pereira B, Billaud M, Almeida R. RNA-binding proteins in cancer: old players and new actors. Trends Cancer. 2017;3:506-28.

8. Wurth L, Gebauer F. RNA-binding proteins, multifaceted translational regulators in cancer. Biochim Biophys Acta. 2015;1849:881-6.

9. Guan R, El-Rass S, Spillane D, Lam S, Wang Y, Wu J, et al. rbm47, a novel RNA binding protein, regulates zebrafish head development. Dev Dyn. 2013;242:1395-404.

10. Fossat N, Radziewic T, Jones V, Tourle K, Tam PP. Conditional restoration and inactivation of Rbm47 reveal its tissue-context requirement for viability and growth. Genesis. 2016;54:115-22.

11. Cieply B, Park JW, Nakauka-Ddamba A, Bebee TW, Guo Y, Shang $\mathrm{X}$, et al. Multiphasic and dynamic changes in alternative splicing during induction of pluripotency are coordinated by numerous RNA-binding proteins. Cell Rep. 2016;15:247-55.

12. Vanharanta S, Marney CB, Shu W, Valiente M, Zou Y, Mele A, et al. Loss of the multifunctional RNA-binding protein RBM47 as a source of selectable metastatic traits in breast cancer. Elife. 2014;3:e02734.

13. Treiber T, Treiber N, Plessmann U, Harlander S, Daiss JL, Eichner N, et al. A compendium of RNA-binding proteins that regulate microRNA biogenesis. Mol Cell. 2017;66:270-84 e13.

14. Fossat N, Tourle K, Radziewic T, Barratt K, Liebhold D, Studdert $\mathrm{JB}$, et al. $\mathrm{C}$ to $\mathrm{U}$ RNA editing mediated by APOBEC1 requires RNA-binding protein RBM47. EMBO Rep. 2014;15:903-10.

15. Wei Y, Zhang F, Zhang Y, Wang X, Xing C, Guo J, et al. Posttranscriptional regulator Rbm47 elevates $\mathrm{IL}-10$ production and promotes the immunosuppression of B cells. Cell Mol Immunol. 2019;16:580-9.

16. Sakurai T, Isogaya K, Sakai $S$, Morikawa M, Morishita $Y$, Ehata $\mathrm{S}$, et al. RNA-binding motif protein 47 inhibits Nrf2 activity to suppress tumor growth in lung adenocarcinoma. Oncogene. 2016;35:5000-9.

17. Rokavec M, Kaller M, Horst D, Hermeking H. Pan-cancer EMTsignature identifies RBM47 down-regulation during colorectal cancer progression. Sci Rep. 2017;7:4687.

18. Puisieux A, Brabletz T, Caramel J. Oncogenic roles of EMTinducing transcription factors. Nat Cell Biol. 2014;16:488-94.

19. Hein MY, Hubner NC, Poser I, Cox J, Nagaraj N, Toyoda Y, et al. A human interactome in three quantitative dimensions organized by stoichiometries and abundances. Cell. 2015;163:712-23.
20. Dutertre M, Lambert S, Carreira A, Amor-Gueret M, Vagner S. DNA damage: RNA-binding proteins protect from near and far. Trends Biochem Sci. 2014;39:141-9.

21. Peters D, Radine C, Reese A, Budach W, Sohn D, Jänicke RU. TheDEAD-box RNA helicase DDX41 is a novel repressor of p21(WAF1/CIP1) mRNA translation. J Biol Chem. 2017;292: 8331-41.

22. Sohn D, Budach W, Jänicke RU. Caspase-2 is required for DNA damage-induced expression of the CDK inhibitor p21(WAF1/ CIP1). Cell Death Differ. 2011;18:1664-74.

23. Jänicke RU, Sprengart ML, Wati MR, Porter AG. Caspase-3 is required for DNA fragmentation and morphological changes associated with apoptosis. J Biol Chem. 1998;273:9357-60.

24. Brenner JC, Graham MP, Kumar B, Saunders LM, Kupfer R, Lyons $\mathrm{RH}$, et al. Genotyping of $73 \mathrm{UM}-\mathrm{SCC}$ head and neck squamous cell carcinoma cell lines. Head Neck. 2010;32:417-26.

25. Drewinko B, Romsdahl MM, Yang LY, Ahearn MJ, Trujillo JM. Establishment of a human carcinoembryonic antigen-producing colon adenocarcinoma cell line. Cancer Res. 1976;36(2 Pt 1): 467-75.

26. Wang S, El-Deiry WS. p73 or p53 directly regulates human p53 transcription to maintain cell cycle checkpoints. Cancer Res. 2006;66:6982-9.

27. Graupner V, Schulze-Osthoff K, Essmann F, Jänicke RU. Functional characterization of p53beta and p53gamma, two isoforms of the tumor suppressor p53. Cell Cycle. 2009;8:1238-48.

28. Debacq-Chainiaux F, Erusalimsky JD, Campisi J, Toussaint O. Protocols to detect senescence-associated beta-galactosidase (SAbetagal) activity, a biomarker of senescent cells in culture and in vivo. Nat Protoc. 2009;4:1798-806.

29. Roberts TC, Hart JR, Kaikkonen MU, Weinberg MS, Vogt PK, Morris KV. Quantification of nascent transcription by bromouridine immunocapture nuclear run-on RT-qPCR. Nat Protoc. 2015;10:1198-211.

30. Meek DW, Anderson CW. Posttranslational modification ofp53: cooperative integrators of function. Cold Spring Harb Perspect Biol. 2009;1:a000950.

31. Karni-Schmidt O, Lokshin M, Prives C. The roles of MDM2 and MDMX in cancer. Annu Rev Pathol. 2016;11:617-44.

32. Sohn D, Essmann F, Schulze-Osthoff K, Jänicke RU. p21 blocks irradiation-induced apoptosis downstream of mitochondria by inhibition of cyclin-dependent kinase-mediated caspase- 9 activation. Cancer Res. 2006;66:11254-62.

33. Jänicke RU, Sohn D, Essmann F, Schulze-Osthoff K. The multiple battles fought by anti-apoptotic p21. Cell Cycle. 2007;6:407-13.

34. Saldana-Meyer R, Recillas-Targa F. Transcriptional and epigenetic regulation of the p53 tumor suppressor gene. Epigenetics. 2011;6:1068-77.

35. Baillat D, Laitem C, Leprivier G, Margerin C, Aumercier M. Ets-1 binds cooperatively to the palindromic Ets-binding sites in the p53 promoter. Biochem Biophys Res Commun. 2009;378:213-7.

36. Suzuki H, Romano-Spica V, Papas TS, Bhat NK. ETS1 suppresses tumorigenicity of human colon cancer cells. Proc Natl Acad Sci USA. 1995;92:4442-6.

37. Ray D, Kazan H, Cook KB, Weirauch MT, Najafabadi HS, Li X, et al. A compendium of RNA-binding motifs for decoding gene regulation. Nature. 2013;499:172-7.

38. Chaudhary R, Lal A. Long noncoding RNAs in the p53 network. Wiley Interdiscip Rev RNA. 2017;8:e1410.

39. Tano K, Onoguchi-Mizutani R, Yeasmin F, Uchiumi F, Suzuki Y, Yada $\mathrm{T}$, et al. Identification of minimal p53 promoter region regulated by MALAT1 in human lung adenocarcinoma cells. Front Genet. 2017;8:208.

40. Zhai N, Xia Y, Yin R, Liu J, Gao F. A negative regulation loop of long noncoding RNA HOTAIR and p53 in non-small-cell lung cancer. Onco Targets Ther. 2016;9:5713-20. 
41. Jänicke RU, Sohn D, Schulze-Osthoff K. The dark side of a tumor suppressor: anti-apoptotic p53. Cell Death Differ. 2008;15:959-76.

42. Pappas K, Xu J, Zairis S, Resnick-Silverman L, Abate F, Steinbach N, et al. p53 maintains baseline expression of multiple tumor suppressor genes. Mol Cancer Res. 2017;15: 1051-62.
43. Kim YE, Won M, Lee SG, Park C, Song CH, Kim KK. RBM47regulated alternative splicing of TJP1 promotes actin stress fiber assembly during epithelial-to-mesenchymal transition. Oncogene. 2019. https://doi.org/10.1038/s41388-019-0892-5. [Epub ahead of print].

44. Rojo de la Vega M, Chapman E, Zhang DD. NRF2 and the hallmarks of cancer. Cancer Cell. 2018;34:21-43. 